\title{
Precise coincidence of effective potentials in the integral and fractional quantum Hall effects
}

\author{
F. Lado \\ Department of Physics, North Carolina State University, Raleigh, NC 27695-8202, USA \\ Received 3 October 2002; accepted 28 March 2003 \\ Communicated by V.M. Agranovich
}

\begin{abstract}
The exact $n$-body distribution functions are calculated for a two-dimensional, noninteracting quantum electron gas in an external magnetic field for any temperature and density. At low temperature and filled lowest-Landau-level, these functions are identical to the exact distribution functions obtained by Jancovici [Phys. Rev. Lett. 46 (1981) 386] for the classical twodimensional one-component plasma at the special plasma parameter $\Gamma=2$, establishing that the quantum state with filling factor $v=1$, associated with the integral quantum Hall effect, is precisely described by an effective classical potential $\phi(r)=-2 \ln r$. Further, this Boltzmann factor exactly matches that constructed by Laughlin [Phys. Rev. Lett. 50 (1983) 1395] to account for the fractional quantum Hall effect.
\end{abstract}

(C) 2003 Elsevier Science B.V. All rights reserved.

A system of noninteracting electrons in a plane subject to an external magnetic field, first studied by Landau [1] in 1930 to account for diamagnetism, became the basis for understanding the integral quantum Hall effect [2], discovered by von Klitzing et al. [3] fifty years later. The discovery of the fractional quantum Hall effect [4] not long after called for a different explanation and an important contribution was made by Laughlin [5], who invoked the classical two-dimensional one-component plasma [6] in constructing his solution. In this Letter, we report a surprising connection between the same two-dimensional one-component plasma (2DOCP) and the integral quantum Hall effect, as modeled by the simplest system, Landau's noninteracting electrons in a plane.

The system consists of $N$ electrons at temperature $T$ in area $A$. A uniform magnetic field $B_{0}$ is applied perpendicular to the plane of $A$. The canonical partition function $Q_{N}$ for this system can be written

$$
Q_{N}=\frac{1}{N ! \Lambda^{2 N}} \int W_{N}\left(\boldsymbol{r}^{N}\right) d \boldsymbol{r}_{1} \cdots d \boldsymbol{r}_{N}
$$

where $W_{N}\left(\boldsymbol{r}^{N}\right)$ is the (antisymmetrized) Slater sum,

$$
W_{N}\left(\boldsymbol{r}^{N}\right)=\Lambda^{2 N} \sum_{P}(-1)^{|P|} \sum_{k} \Psi_{k}^{*}\left(P \boldsymbol{r}^{N}\right) e^{-\beta \mathcal{H}} \Psi_{k}\left(\boldsymbol{r}^{N}\right),
$$

E-mail address: fred_lado@ncsu.edu (F. Lado). 
which plays the role of Boltzmann factor. Here the $\Psi_{k}$ are a complete set of $N$-particle states distinguished by index $k$ representing $2 N$ quantum numbers. The sum over permutations $P$, with even or odd parity $|P|$ and antisymmetric weighting, accounts for the Fermi-Dirac statistics of the indistinguishable electrons; the thermal de Broglie wavelength $\Lambda$ serves to make the Slater sum dimensionless but plays no other role in the sequel. The Hamiltonian $\mathcal{H}$ for noninteracting electrons in an external magnetic field is $\mathcal{H}=\sum_{j=1}^{N}\left[-i \hbar \nabla_{j}+e \boldsymbol{A}\left(\boldsymbol{r}_{j}\right)\right]^{2} / 2 m_{e}$, with $m_{e}$ the electron mass, $-e$ its charge, and $\boldsymbol{A}(\boldsymbol{r})$ a vector potential that yields a uniform magnetic field $\boldsymbol{B}_{0}=\nabla \times \boldsymbol{A}(\boldsymbol{r})$, which we shall take to define the $z$ direction. The system is taken to be fully spin-polarized, so that spin degrees of freedom may be ignored.

Since interactions between electrons are being neglected, the Schrödinger equation for the energy eigenstates is readily solved and the sum over states in Eq. (2) can be carried out. The result is [7]

$$
\begin{aligned}
W_{N}\left(\boldsymbol{r}^{N}\right)= & \left(\Lambda^{2} / 4 \pi l_{0}^{2} \sinh \beta \mu_{B} B_{0}\right)^{N} \\
& \times \sum_{P}(-1)^{|P|} \exp \left\{-\sum_{j=1}^{N}\left[\left(\boldsymbol{r}_{j}-P \boldsymbol{r}_{j}\right)^{2} / 4 l_{0}^{2} \tanh \beta \mu_{B} B_{0}-i\left(x_{j} P y_{j}-y_{j} P x_{j}\right) / 2 l_{0}^{2}\right]\right\},
\end{aligned}
$$

where $\boldsymbol{r}_{j}$ locates electron $j$ in the $x y$ plane, $\mu_{B}=e \hbar / 2 m_{e}$ is the Bohr magneton, and $l_{0}=\left(\hbar / e B_{0}\right)^{1 / 2}$ the characteristic magnetic length.

With the Slater sum in hand, we seek to determine the $n$-body distribution function

$$
\rho_{N}^{(n)}\left(\boldsymbol{r}_{1}, \ldots, \boldsymbol{r}_{n}\right)=\frac{1}{(N-n) ! \Lambda^{2 N} Q_{N}} \int W_{N}\left(\boldsymbol{r}^{N}\right) d \boldsymbol{r}_{n+1} \cdots d \boldsymbol{r}_{N}
$$

for any $n$. Now, each permutation $P$ in Eq. (3) produces a disjoint set of ring-type integrands, with each particle appearing in one and only one ring. Particle positions $1,2, \ldots, n$ are not integrated out and will be called root points. The remaining $N-n$ particle positions are variables of integration, to be called field points. The bond linking adjacent particles $i$ and $j$ in a ring is

$$
f\left(\boldsymbol{r}_{i}, \boldsymbol{r}_{j}\right)=\left(4 \pi l_{0}^{2} \sinh \beta \mu_{B} B_{0}\right)^{-1} \exp \left[-\left(\boldsymbol{r}_{i}-\boldsymbol{r}_{j}\right)^{2} / 4 l_{0}^{2} \tanh \beta \mu_{B} B_{0}+i\left(x_{i} y_{j}-y_{i} x_{j}\right) / 2 l_{0}^{2}\right] .
$$

Let $I_{k}\left(\boldsymbol{r}_{i}, \boldsymbol{r}_{j}\right)$ be a chain of links beginning at root point $\boldsymbol{r}_{i}$ and ending at root point $\boldsymbol{r}_{j}$ with $k$ field points in between; i.e., $I_{k}\left(\boldsymbol{r}_{i}, \boldsymbol{r}_{j}\right)=\int f\left(\boldsymbol{r}_{i}, \boldsymbol{r}_{n_{1}}\right) f\left(\boldsymbol{r}_{n_{1}}, \boldsymbol{r}_{n_{2}}\right) \cdots f\left(\boldsymbol{r}_{n_{k}}, \boldsymbol{r}_{j}\right) d \boldsymbol{r}_{n_{1}} \cdots d \boldsymbol{r}_{n_{k}}$. The canonical distribution function $\rho_{N}^{(n)}\left(\boldsymbol{r}_{1}, \ldots, \boldsymbol{r}_{n}\right)$ then becomes [8]

$$
\begin{aligned}
\rho_{N}^{(n)}\left(\boldsymbol{r}_{1}, \ldots, \boldsymbol{r}_{n}\right)= & \frac{1}{(N-n) ! Q_{N}} \sum_{P}(-1)^{|P|} \sum_{m=0}^{N-n}(-1)^{m} \frac{(N-n) !}{(N-n-m) !} \\
& \times \sum_{k_{1}, k_{2}, \ldots, k_{n}} \delta\left(m, \sum_{j} k_{j}\right) I_{k_{1}}\left(\boldsymbol{r}_{1}, P \boldsymbol{r}_{1}\right) I_{k_{2}}\left(\boldsymbol{r}_{2}, P \boldsymbol{r}_{2}\right) \cdots \\
& \times I_{k_{n}}\left(\boldsymbol{r}_{n}, P \boldsymbol{r}_{n}\right)(N-n-m) ! Q_{N-n-m} .
\end{aligned}
$$

Now the permutations $P$ are among the $n$ root points only, while $m$ is the total number of field points in the rooted rings. The sums over number of field points $k_{j}$ in individual chains are collectively constrained by the requirement $\sum_{j=1}^{n} k_{j}=m$, expressed by the Kronecker delta $\delta(i, j)$ in the summand. This constraint can be removed, and the individual sums over the $k_{j}$ factored, by transforming these expressions from particle number $N$ to chemical potential $\mu$ as independent variable using the grand canonical formalism. We have then $\Xi=\sum_{N=0}^{\infty} e^{\beta \mu N} Q_{N}$ for the partition function and $\rho^{(n)}\left(\boldsymbol{r}_{1}, \ldots, \boldsymbol{r}_{n}\right)=\Xi^{-1} \sum_{N=n}^{\infty} e^{\beta \mu N} Q_{N} \rho_{N}^{(n)}\left(\boldsymbol{r}_{1}, \ldots, \boldsymbol{r}_{n}\right)$ for the $n$-body distribution function. Using Eq. (6) in the latter we now get simply

$$
\rho^{(n)}\left(\boldsymbol{r}_{1}, \ldots, \boldsymbol{r}_{n}\right)=\sum_{P}(-1)^{|P|} \prod_{j=1}^{n}\left[\sum_{k=0}^{\infty}(-1)^{k} e^{(k+1) \beta \mu} I_{k}\left(\boldsymbol{r}_{j}, P \boldsymbol{r}_{j}\right)\right] \text {. }
$$


Starting from $I_{0}\left(\boldsymbol{r}_{i}, \boldsymbol{r}_{j}\right)=f\left(\boldsymbol{r}_{i}, \boldsymbol{r}_{j}\right)$, one finds by induction that

$$
\begin{aligned}
I_{k}\left(\boldsymbol{r}_{i}, \boldsymbol{r}_{j}\right)= & {\left[4 \pi l_{0}^{2} \sinh \left((k+1) \beta \mu_{B} B_{0}\right)\right]^{-1} } \\
& \times \exp \left[-\left(\boldsymbol{r}_{i}-\boldsymbol{r}_{j}\right)^{2} / 4 l_{0}^{2} \tanh \left((k+1) \beta \mu_{B} B_{0}\right)+i\left(x_{i} y_{j}-y_{i} x_{j}\right) / 2 l_{0}^{2}\right],
\end{aligned}
$$

so that finally

$$
\rho^{(n)}\left(\boldsymbol{r}_{1}, \ldots, \boldsymbol{r}_{n}\right)=\sum_{P}(-1)^{|P|} \prod_{j=1}^{n}\left\{\eta\left(\left|\boldsymbol{r}_{j}-P \boldsymbol{r}_{j}\right|\right) \exp \left[i\left(x_{j} P y_{j}-y_{j} P x_{j}\right) / 2 l_{0}^{2}\right]\right\},
$$

with

$$
\eta(r)=\frac{1}{4 \pi l_{0}^{2}} \sum_{j=1}^{\infty} \frac{(-1)^{j-1} e^{j \beta \mu}}{\sinh \left(j \beta \mu_{B} B_{0}\right)} \exp \left[-r^{2} / 4 l_{0}^{2} \tanh \left(j \beta \mu_{B} B_{0}\right)\right] .
$$

This key result can be rewritten in a form that more directly displays the familiar Fermi degeneracy at low temperatures. We use the generating function of Laguerre polynomials, $\exp [-x z /(1-z)] /(1-z)=$ $\sum_{k=0}^{\infty} L_{k}(x) z^{k}$, with $x=r^{2} / 2 l_{0}^{2}$ and $z=\exp \left(-2 j \beta \mu_{B} B_{0}\right)$ in Eq. (10) and then sum over $j$ to get

$$
\eta(r)=\frac{e^{-r^{2} / 4 l_{0}^{2}}}{2 \pi l_{0}^{2}} \sum_{k=0}^{\infty} \frac{L_{k}\left(r^{2} / 2 l_{0}^{2}\right)}{1+e^{-\beta\left[\mu-(2 k+1) \mu_{B} B_{0}\right]}},
$$

where now the summation is over Landau levels, of energy $\epsilon_{k}=(2 k+1) \mu_{B} B_{0}$.

The first of the distribution functions $(9), \rho^{(1)}\left(\boldsymbol{r}_{1}\right)=\eta(0)$, is just the uniform density $\rho=\bar{N} / A$ expressed in terms of the chemical potential $\mu$,

$$
\rho=\frac{1}{2 \pi l_{0}^{2}} \sum_{k=0}^{\infty} \frac{1}{1+e^{-\beta\left[\mu-(2 k+1) \mu_{B} B_{0}\right]}} .
$$

For low temperatures and large magnetic fields, i.e., for $\beta \mu_{B} B_{0} \gg 1$, the summand in Eq. (12) acts like a discrete unit step function, so that for (integer) $n$ filled Landau levels one has $2 \pi \rho l_{0}^{2}=n$.

The dimensionless forms $g^{(n)}\left(\boldsymbol{r}_{1}, \ldots, \boldsymbol{r}_{n}\right) \equiv \rho^{(n)}\left(\boldsymbol{r}_{1}, \ldots, \boldsymbol{r}_{n}\right) / \rho^{n}$ are preferable in writing out higher distribution functions. Explicitly, we have for the next three,

$$
\begin{aligned}
& g^{(2)}\left(\boldsymbol{r}_{1}, \boldsymbol{r}_{2}\right)=1- D^{2}\left(r_{12}\right), \\
& g^{(3)}\left(\boldsymbol{r}_{1}, \boldsymbol{r}_{2}, \boldsymbol{r}_{3}\right)=1- {\left[D^{2}\left(r_{12}\right)+D^{2}\left(r_{13}\right)+D^{2}\left(r_{23}\right)\right] } \\
&+ 2 D\left(r_{12}\right) D\left(r_{23}\right) D\left(r_{31}\right) \cos \left(\left|\boldsymbol{r}_{12} \times \boldsymbol{r}_{13}\right| / 2 l_{0}^{2}\right), \\
& g^{(4)}\left(\boldsymbol{r}_{1}, \boldsymbol{r}_{2}, \boldsymbol{r}_{3}, \boldsymbol{r}_{4}\right)=1-\left[D^{2}\left(r_{12}\right)+\cdots\right](6 \text { terms })+\left[D^{2}\left(r_{12}\right) D^{2}\left(r_{34}\right)+\cdots\right](3 \text { terms }) \\
&+2\left[D\left(r_{12}\right) D\left(r_{23}\right) D\left(r_{31}\right) \cos \left(\left|\boldsymbol{r}_{12} \times \boldsymbol{r}_{13}\right| / 2 l_{0}^{2}\right)+\cdots\right](4 \text { terms }) \\
&-2\left[D\left(r_{12}\right) D\left(r_{23}\right) D\left(r_{34}\right) D\left(r_{41}\right) \cos \left(\left|\boldsymbol{r}_{13} \times \boldsymbol{r}_{24}\right| / 2 l_{0}^{2}\right)+\cdots\right](3 \text { terms }),
\end{aligned}
$$

where $D(r)=\eta(r) / \eta(0)$.

At this point we note a remarkable fact. For physical conditions such that this noninteracting quantum electron system just fills the lowest Landau level, i.e., for $\beta \mu_{B} B_{0} \gg 1$ and $2 \pi \rho l_{0}^{2}=1$, so that $D(r)=\exp \left(-\pi \rho r^{2} / 2\right)$, the exact distribution functions $g^{(n)}\left(\boldsymbol{r}_{1}, \ldots, \boldsymbol{r}_{n}\right)$ displayed above are identical to the exact $g^{(n)}\left(\boldsymbol{r}_{1}, \ldots, \boldsymbol{r}_{n}\right)$ of a classical two-dimensional one-component plasma obtained by Jancovici [9] for the plasma parameter value $\Gamma=2$. Working backwards, we may then conclude that the quantum state for the filled lowest-Landau-level is precisely described by an effective classical pair potential $\phi(r)=-2 \ln \left(r / l_{0}\right)$. This is confirmed below by analytic 
calculation. More generally, we can calculate effective potentials for a low-temperature system with $n>1$ Landau levels filled; their long-range form is $\phi(r) \sim-(2 / n) \ln \left(r / l_{0}\right)$.

Having found that the filled $n=1$ state at zero temperature is exactly described by an effective pair potential, we continue by assuming that, in general, there exists a pair function $\phi(r)$ such that the Slater sum, Eq. (2), may be written $W_{N}\left(\boldsymbol{r}^{N}\right) \approx \exp \left[-\sum_{i<j} \phi\left(r_{i j}\right)\right]$, a notion first discussed by Uhlenbeck and Gropper [11,12]. Given that assumption, the Yvon-Born-Green (YBG) hierarchy [10] then offers an exact route to such a function [8]. The second member of this hierarchy reads

$$
\nabla_{1} \ln g^{(2)}\left(\boldsymbol{r}_{1}, \boldsymbol{r}_{2}\right)=-\nabla_{1} \phi\left(r_{12}\right)-\rho \int d \boldsymbol{r}_{3} \nabla_{1} \phi\left(r_{13}\right) \frac{g^{(3)}\left(\boldsymbol{r}_{1}, \boldsymbol{r}_{2}, \boldsymbol{r}_{3}\right)}{g^{(2)}\left(\boldsymbol{r}_{1}, \boldsymbol{r}_{2}\right)}
$$

where now $\phi(r)$ is the only unknown. Dotting $\hat{\boldsymbol{r}}_{12}$ into Eq. (16) and using the substitutions $u(r)=-d \phi(r) / d r$, $v(r)=d \ln g(r) / d r$, and

$$
W(r, s)=\frac{1}{\pi} \int_{0}^{\pi} d \theta \cos \theta\left\{D^{2}(t)-2 D(r) D(s) D(t) \cos \left[\left(r s / 2 l_{0}^{2}\right) \sin \theta\right]\right\},
$$

with $\cos \theta=\hat{\boldsymbol{r}} \cdot \hat{\boldsymbol{s}}$ and $t=\left(r^{2}+s^{2}-2 r s \cos \theta\right)^{1 / 2}$, Eq. (16) becomes an integral equation,

$$
u(r)=v(r)+\frac{2 \pi \rho}{g(r)} \int_{0}^{\infty} d s s u(s) W(r, s),
$$

that can be solved iteratively for $u(r)$; a final integration yields the effective potential. Here we have dropped those terms of $g^{(3)}\left(\boldsymbol{r}_{1}, \boldsymbol{r}_{2}, \boldsymbol{r}_{3}\right)$ that do not contribute because of symmetry and have omitted the superscript on the pair function for simplicity.

For high-temperature states, this straightforward procedure works well. The effective potentials found in this way for states with $\beta \mu_{B} B_{0}=1$ and reduced densities $2 \pi \rho l_{0}^{2}=1,2,3,4,5$ are shown in Fig. 1 . As the density increases the amplitude of the effective potential decreases, but the changes are not large.

When applied at low temperatures, however, this simple procedure becomes problematical, as divergences make an appearance. Their source is not hard to find. Expanding the Bessel function $J_{0}(k r)$ and evaluating term by term, we get for the Fourier transform of $h(r)=g(r)-1$,

$$
\rho \tilde{h}(k)=2 \pi \rho \int_{0}^{\infty} d r r h(r) J_{0}(k r)=-1+\frac{1}{2}\left(k l_{0}\right)^{2}+\cdots
$$

for $\beta \mu_{B} B_{0} \gg 1$ and $2 \pi \rho l_{0}^{2}=n$. Then $S(0)=1+\rho \tilde{h}(0)=0$, the compressibility $\chi_{T}=\beta S(0) / \rho$ vanishes, and the transform of the direct correlation function $c(r)[10], \rho \tilde{c}(k)=\rho \tilde{h}(k) / 1+\rho \tilde{h}(k)$, acquires a $k^{-2}$ divergence at the origin. The long-range behavior of $\phi(r)$ follows that of $-c(r)$ and so $\rho \tilde{\phi}(k) \approx 2 /\left(k l_{0}\right)^{2}$ for small $k$, independent of density, and we finally get the asymptotic forms $\phi(r) \sim-(2 / n) \ln \left(r / l_{0}\right)$ and $u(r) \sim 2 / n r$.

These are troublesome functions for any numerical procedure restricted to a finite range. Fortunately, it turns out that, in this low-temperature regime, many needed integrals can be evaluated analytically, beginning with $W(r, s)$ [7]. Even with analytic kernels, however, Eq. (18) cannot be handled within a finite numerical range without first analytically extracting the asymptotic (LR) part, $u^{\mathrm{LR}}(r)=2 / n r$. The resulting equation for just the short-range (SR) part, $u^{\mathrm{SR}}(r)=u(r)-u^{\mathrm{LR}}(r)$, can then be solved numerically (analytically for $\left.n=1\right)$.

The computed results for reduced densities $2 \pi \rho l_{0}^{2}=1,2,3$ at $\beta \mu_{B} B_{0}=50$ are shown in Fig. 2 as $r u(r)$; for $n=1$, the solution is a constant, $r u(r)=2$, as expected, while for $n=2$ and $3, r u(r)$ makes a smooth transition from 2 at the origin to its long-range constant value of $2 / n$ over a distance $r \approx 5 l_{0}$, by which point particle pairs are essentially uncorrelated. Fig. 3 displays the effective potentials $\phi(r)$ obtained from these $u(r)$. 


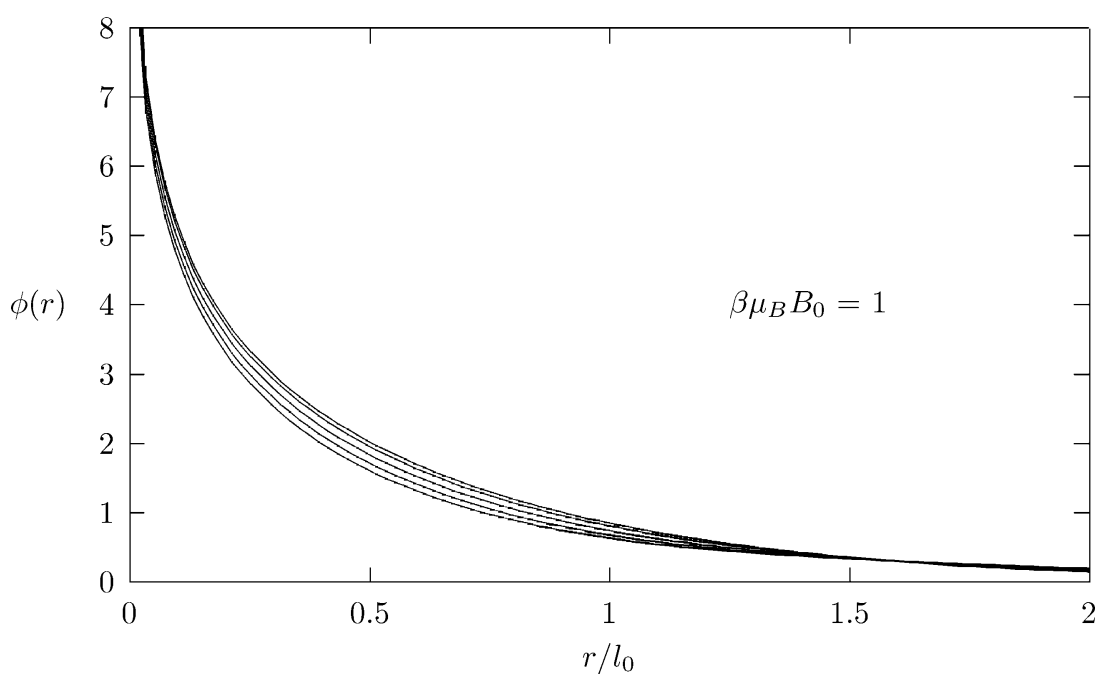

Fig. 1. Effective potentials obtained from the YBG equation at a high temperature and reduced densities $2 \pi \rho l_{0}^{2}=1,2,3,4,5$, read from top to bottom.

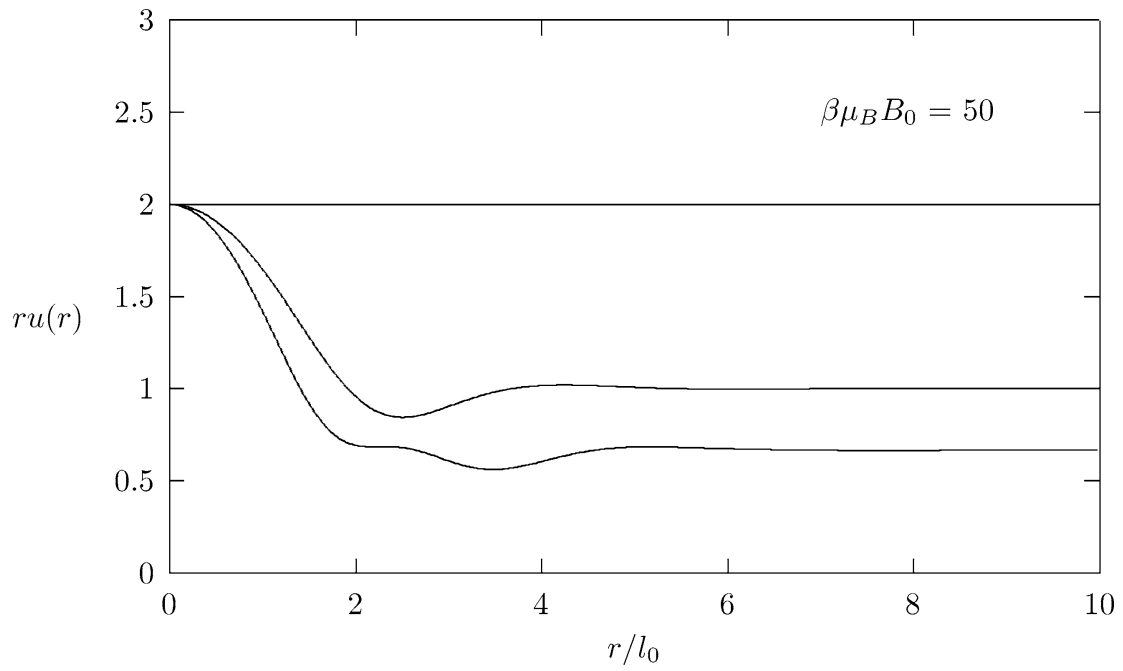

Fig. 2. Logarithmic derivative of the effective potentials, $r u(r)=-d \phi(r) / d \ln r$, obtained from the YBG equation at a low temperature and reduced densities $2 \pi \rho l_{0}^{2}=1,2,3$, read from top to bottom.

The defining potential of the 2DOCP is $w(r)=-\Gamma \ln \left(r / l_{0}\right)$ [6], where $\Gamma$ is the plasma parameter. In this Letter, we have found that the two-dimensional one-component plasma interaction functions as an effective potential $\phi(r)$ for the simple quantum model of noninteracting electrons in a plane, subject to an external magnetic field, when they are taken to low temperatures and form incompressible states. This potential model is exact, $\phi(r)=-2 \ln \left(r / l_{0}\right)$, for filling factor $v=2 \pi \rho l_{0}^{2}=1$, and asymptotic, $\phi(r) \sim-(2 / n) \ln \left(r / l_{0}\right)$, for filling factors $v=n>1$. Those physical conditions, $\beta \mu_{B} B_{0} \gg 1$ and $2 \pi \rho l_{0}^{2}=n$, apply to the integral quantum Hall effect, and so we find that the integral quantum Hall effect maps into the two-dimensional one-component plasma through an 


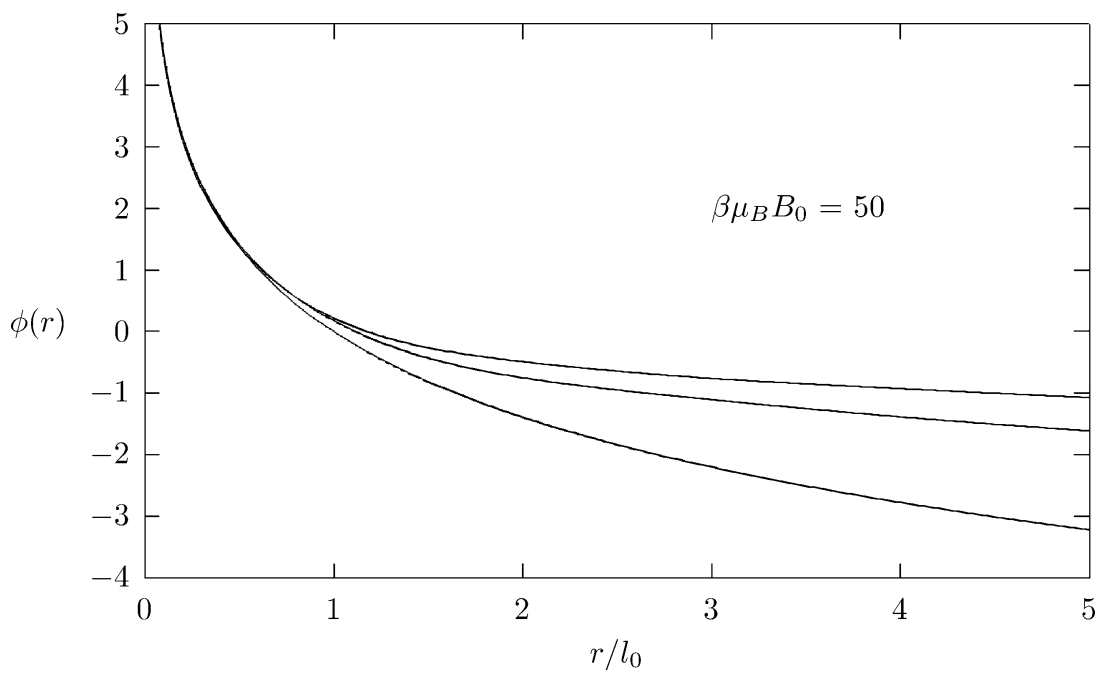

Fig. 3. Effective potentials obtained from the YBG equation at a low temperature and reduced densities $2 \pi \rho l_{0}^{2}=1,2,3$, read from bottom to top.

effective Boltzmann factor

$$
W_{N}^{(1)}\left(\boldsymbol{r}^{N}\right)=\prod_{i<j}\left(\frac{r_{i j}}{l_{0}}\right)^{2} \exp \left[-\frac{1}{2} \sum_{i}\left(\frac{r_{i}}{l_{0}}\right)^{2}\right],
$$

for $v=1$, and, at large interparticle separations $r_{i j}$,

$$
W_{N}^{(n)}\left(\boldsymbol{r}^{N}\right) \sim \prod_{i<j}\left(\frac{r_{i j}}{l_{0}}\right)^{2 / n} \exp \left[-\frac{1}{2} \sum_{i}\left(\frac{r_{i}}{l_{0}}\right)^{2}\right],
$$

for $v=n>1$. Here we have adapted to the present notation the total potential $V$ quoted by Jancovici [9] for 2DOCP particles confined to a disk of radius $R ; r_{i}$ is the radial distance from the disk center.

It is of course well known that the 2DOCP has previously been invoked by Laughlin [5] to construct an $\mathrm{N}$ particle wave function $\psi_{m}$ that accounts for the principal features of the fractional quantum Hall effect. Expressed as an equivalent Boltzmann factor $W=\left|\psi_{m}\right|^{2}$, Laughlin's solution is

$$
W_{N}^{(1 / m)}\left(\boldsymbol{r}^{N}\right)=\prod_{i<j}\left(\frac{r_{i j}}{l_{0}}\right)^{2 m} \exp \left[-\frac{1}{2} \sum_{i}\left(\frac{r_{i}}{l_{0}}\right)^{2}\right],
$$

with $m$ an odd integer, including one.

The coincidence of form in these effective Boltzmann factors for the integral and fractional quantum Hall effects is remarkable. Eq. (20) in particular is the precise intersection of the integral quantum Hall effect, the fractional quantum Hall effect, and the classical two-dimensional one-component plasma.

\section{References}

[1] L.D. Landau, Z. Phys. 64 (1930) 629.

[2] T. Chakraborty, P. Pietiläinen, The Quantum Hall Effects, Springer-Verlag, Berlin, 1995.

[3] K. von Klitzing, G. Dorda, M. Pepper, Phys. Rev. Lett. 45 (1980) 494. 
[4] D.C. Tsui, H.L. Störmer, A.C. Gossard, Phys. Rev. Lett. 48 (1982) 1559.

[5] R.B. Laughlin, Phys. Rev. Lett. 50 (1983) 1395.

[6] J.M. Caillol, D. Levesque, J.J. Weis, J.P. Hansen, J. Stat. Phys. 28 (1982) 325.

[7] F. Lado, Phys. Rev. B, submitted for publication.

[8] F. Lado, J. Chem. Phys. 47 (1967) 5369.

[9] B. Jancovici, Phys. Rev. Lett. 46 (1981) 386.

[10] J.P. Hansen, I.R. McDonald, Theory of Simple Liquids, Academic Press, London, 1986.

[11] G.E. Uhlenbeck, L. Gropper, Phys. Rev. 41 (1932) 79.

[12] K. Huang, Statistical Mechanics, Wiley, New York, 1987, Chapter 9. 